\title{
MicroRNA-related polymorphisms in apoptosis pathway genes are predictive of clinical outcome in patients with limited disease small cell lung cancer
}

\author{
Wei Jiang ${ }^{1}$, Nan $B^{1}{ }^{1}$, Wen-Jue Zhang ${ }^{1}$, Li-Hong $\mathrm{Wu}^{1}{ }^{1}$, Li-Pin Liu ${ }^{1}$, Yu Men ${ }^{1}$, Jing-Bo \\ Wang $^{1}$, Jun Liang ${ }^{1}$, Zhou-Guang Hui ${ }^{1}$, Zong-Mei Zhou ${ }^{1}$, Lu-Hua Wang ${ }^{1}$ \\ ${ }^{1}$ Department of Radiation Oncology, Cancer Hospital, Chinese Academy of Medical Sciences and Peking Union Medical \\ College, Beijing, China
}

Correspondence to: Lu-Hua Wang, e-mail: wlhwq@yahoo.com

Keywords: small cell lung cancer, limited-disease, single nucleotide polymorphisms, microRNA, apoptosis pathway

Received: November 26, 2015

Accepted: February 21, 2016

Published: March 16, 2016

\section{ABSTRACT}

We examined the impact of single nucleotide polymorphisms (SNPs) at miRNA binding sites in the $3^{\prime}$-UTRs of genes in the apoptosis pathway on the prognosis of patients with limited disease-small cell lung cancer (LD-SCLC). Twelve tagSNPs in seven genes were genotyped using blood samples from 146 LD-SCLC patients treated with chemoradiotherapy. Cox proportional hazard regression models and recursive partitioning analysis were performed to identify SNPs significantly associated with overall survival. Three SNPs, CASP8: rs1045494 (C > T), PIK3R1: rs3756668 (A > G) and CASP7: rs4353229 ( $>C$ ), were associated with longer overall survival in LDSCLC patients after chemoradiotherapy. The adjusted hazard ratios ( $95 \%$ confidence intervals) were $0.480(0.258-0.894), 0.405(0.173-0.947)$ and $0.446(0.247-0.802)$, respectively, and remained significant after multiple comparison correction. Moreover, subset analysis showed these SNPs were still predictive of overall survival in stage III patients. Recursive partitioning analysis enabled patients to be classified into three risk subgroups based on unfavorable genotype combinations of the rs1045494 and rs4353229 SNPs. These findings suggest miRNA-related polymorphisms in the apoptosis pathway may be useful biomarkers for selection of LD-SCLC patients likely to benefit from chemoradiotherapy.

\section{INTRODUCTION}

Small cell lung cancer (SCLC), which accounts for approximately $15 \%$ of primary lung cancers [1], is a very aggressive neuroendocrine malignancy characterized by early metastasis and a poor prognosis. Combined chemoradiotherapy (CRT) is administered to the one-third of SCLC patients who present with limited disease (LDSCLC) at diagnosis [2]. However, their prognosis remains poor. There is also substantial inter-individual variation in the clinical response to treatment, which suggests genetic variation plays an important role in determining patients likely to benefit from CRT.

SCLC is characterized by a short doubling time and fast growth, and almost no apoptotic cells are detected in tumor specimens [3]. Cisplatin-based regimens are the standard chemotherapy for SCLC. Cisplatin would be expected to activate apoptotic signaling and kill cells. Likewise, radiotherapy also works by damaging DNA and inducing apoptosis [4]. Altered apoptotic signaling is thought to contribute to tumorigenesis and progression [5], and genetic variants in the apoptotic pathway are reportedly associated with prognosis and treatment strategy in several cancers [5-7], including SCLC [8]. MicroRNAs (miRNAs), a class of small noncoding RNAs, are key regulators of gene expression in many biological processes [9]. Single nucleotide polymorphisms (SNPs) located in the 3'-UTR of miRNA target genes may affect the actions of miRNAs at oncogenes and tumor suppressor genes [10]. However, few published studies have considered the impact of SNPs at miRNA-binding sites in apoptosis pathways and their relationship to SCLC outcomes. We therefore analyzed the effect of variations 
at miRNA-binding sites in apoptosis pathways on the prognosis of LD-SCLC patients.

\section{RESULTS}

\section{Clinical characteristics}

Ultimately, 146 patients who received curative chemoradiotherapy for LD-SCLC were included in the study. Of those, $91.8 \%$ were diagnosed at stage III. EP (etoposide + cisplatin) and EC (etoposide + carboplatin) were the most common regimens. Chemotherapy was delivered concurrently or sequentially. Prophylactic cranial irradiation (PCI) was administered to $43.2 \%$ of patients. In $90.5 \%$ of those, the primary lesion showed a complete remission (CR) or a partial response (PR). The overall median survival time (MST) and 5-year overall survival (OS) rate were 35.1 months and $38.9 \%$, respectively. The median follow up time was 42.2 months. Age, Karnofsky performance score (KPS), Charison comorbidity index (CCI) and PCI were all significantly associated with the survival outcome ( $p<0.05$; log-rank test). The clinical characteristics of the patients are summarized in Table 1.

\section{Association of individual SNPs with overall survival}

Three SNPs were significantly associated with OS, and the association remained significant after adjusting for age, gender, KPS, smoking history, CCI and PCI (Table 2). One variant of CASP8 (HGNC:1509), rs1045494, had a $48.6 \%$ lower risk of death (adjusted hazard ratio $[\mathrm{HR}]=0.514,95 \%$ confidence interval [CI]: $0.287-$ 0.921). In addition, the variant homozygous genotype of rs4353229 in CASP7 (HGNC:1508) was associated with a 2.245 -fold increase in the risk of death (adjusted $\mathrm{HR}=2.245,95 \%$ CI: 1.247-4.041), while rs3756668 in phosphoinositide-3-kinase regulatory subunit 1 (alpha) (PIK3R1, HGNC:8979) was associated with a $57.9 \%$ decrease in the risk of death (adjusted HR $=0.421,95 \%$ CI: 0.190-0.931) (Figure 1A-1C). After multiple comparison correction, these SNPs still had a $q<0.05$. Two other SNPs, rs12755 in PIK3R1 and rs13429049 in BCL2-like 11 (apoptosis facilitator) (BCL2L11, HGNC:994), were also significantly associated with OS (Supplementary Table S1).

\section{Recursive partitioning analysis (RPA)}

Five SNPs significantly associated with OS after multiple comparisons $(q<0.05)$ (rs1045494 in a dominant model and rs12755, rs13429049, rs3756668 and rs4353229 in recessive models) were included in a recursive partitioning analysis of the effect of unfavorable genotype combinations. Rs1045494 and rs4353229 were eventually selected to develop a RPA model that enabled us to split the data set into three risk classifications (Table 3). The 5-year OS rates were $60.3 \%, 28.4 \%$ and $24.1 \%$ in the low-, intermediate- and high-risk classes, respectively (Figure 1D).

\section{Association of individual SNPs with OS in stage III patients}

In the 134 patients with stage III SCLC, the overall MST and 5-year OS rate were 35.1 months and 38.2\%, respectively. Rs1045494, rs3756668 and rs4353229 remained the three SNPs associated with survival (Supplementary Table S2). The associated HRs adjusted for clinical covariates were 0.480, 0.405 and 2.316, respectively. After correcting for multiple comparisons, genetic variants of rs1045494 and rs4353229 continued to exhibit significant prognostic value, while the association of rs3756668 was borderline.

\section{Validation of the RPA classification using bootstrap analysis}

The RPA predictive model based on SNPs in the apoptosis pathway was internally validated using bootstrap analysis with a 1000-resample set. The differences in OS among the RPA classes were significant ( $p<0.0001$; logrank test). The concordance probability estimate (CPE) used to quantify the RPA classification's discrimination demonstrated good performance in the resampling internal validation $(0.71)$ datasets.

\section{DISCUSSION}

Apoptosis, or programmed cell death, is a fundamental process of controlled cell elimination essential for cellular homeostasis in multicellular organisms [11]. There are two principal interconnected signaling pathways that respectively control apoptosis by activating caspases via the $\mathrm{Bcl}-2$ family and death receptor-related factors [12]. Expression of variants of the genes involved can lead to apoptotic dysregulation that contributes to the development of cancer [13] or is associated with cancer susceptibility and progression [14-16].

In this study, we identified three SNPs at miRNA binding sites on target genes in the apoptosis pathway that affected the OS of LD-SCLC as well as stage III SCLC patients who underwent curative chemoradiotherapy. Moreover, the unfavorable genotype combination of CASP8: rs1045494 and CASP7: rs4353229 amplified the effect of the individual polymorphisms and were of greater potential predictive value for LD-SCLC patients. This is the first study evaluating the combined effect of polymorphisms interacting with apoptosis in SCLC. 
Table 1: Clinical characteristics of patients with limited disease-small cell lung cancer

\begin{tabular}{|c|c|c|c|c|}
\hline Variables & & $N(\%)$ & $5 y$ OS & $P$ \\
\hline \multirow[t]{2}{*}{ Gender } & Male & $104(71.2 \%)$ & $33.0 \%$ & \multirow[t]{2}{*}{0.065} \\
\hline & Female & $42(28.8 \%)$ & $47.2 \%$ & \\
\hline \multirow[t]{2}{*}{ Age } & $\leq 60$ & $95(65.1 \%)$ & $43.0 \%$ & \multirow[t]{2}{*}{0.015} \\
\hline & $>60$ & $51(34.9 \%)$ & $30.8 \%$ & \\
\hline \multirow[t]{2}{*}{ KPS } & $\geq 90$ & $61(41.8 \%)$ & $47.5 \%$ & \multirow[t]{2}{*}{0.009} \\
\hline & $<90$ & $85(58.2 \%)$ & $33.1 \%$ & \\
\hline \multirow[t]{2}{*}{ Location } & Left lobe & $69(47.3 \%)$ & $39.3 \%$ & \multirow[t]{2}{*}{0.987} \\
\hline & Right lobe & $77(52.7 \%)$ & $37.8 \%$ & \\
\hline \multirow[t]{2}{*}{ Smoking } & Yes & $96(65.8 \%)$ & $35.7 \%$ & \multirow[t]{2}{*}{0.088} \\
\hline & No & $50(34.2 \%)$ & $40.1 \%$ & \\
\hline \multirow[t]{3}{*}{ Charison comorbidity index } & $\leq 3$ & $130(89.0 \%)$ & $41.7 \%$ & \multirow[t]{3}{*}{0.030} \\
\hline & $4-5$ & $13(8.9 \%)$ & $19.5 \%$ & \\
\hline & $6-7$ & $3(2.1 \%)$ & $0 \%$ & \\
\hline \multirow[t]{2}{*}{ Weight loss } & With & $27(18.5 \%)$ & $51.3 \%$ & \multirow[t]{2}{*}{0.393} \\
\hline & Without & $119(81.5 \%)$ & $35.2 \%$ & \\
\hline \multirow[t]{6}{*}{ AJCC stage } & I A & $1(0.7 \%)$ & $100.0 \%$ & \multirow[t]{6}{*}{0.573} \\
\hline & I B & $2(1.4 \%)$ & $0 \%$ & \\
\hline & II A & $5(3.4 \%)$ & NA & \\
\hline & II B & $4(2.7 \%)$ & NA & \\
\hline & III A & $68(46.6 \%)$ & $41.7 \%$ & \\
\hline & III B & $66(45.2 \%)$ & $34.9 \%$ & \\
\hline \multirow[t]{2}{*}{ Treatment modality } & Concurrent & $80(54.8 \%)$ & $46.8 \%$ & \multirow[t]{2}{*}{0.401} \\
\hline & Sequential & $66(45.2 \%)$ & $31.2 \%$ & \\
\hline \multirow[t]{3}{*}{ Chemotherapy cycles } & $<4$ & $9(6.2 \%)$ & NA & \multirow[t]{3}{*}{0.382} \\
\hline & $4-6$ & $126(86.3 \%)$ & $40.2 \%$ & \\
\hline & $>6$ & $11(7.5 \%)$ & $38.6 \%$ & \\
\hline \multirow[t]{2}{*}{ Radiotherapy dose } & $<60$ & $46(30.7 \%)$ & $39.3 \%$ & \multirow[t]{2}{*}{0.525} \\
\hline & $\geq 60$ & $100(69.3 \%)$ & $39.9 \%$ & \\
\hline \multirow[t]{2}{*}{ PCI } & With & $63(43.2 \%)$ & $62.1 \%$ & \multirow[t]{2}{*}{$1.54 \mathrm{E}-4$} \\
\hline & Without & $83(56.8 \%)$ & $23.1 \%$ & \\
\hline
\end{tabular}

Abbreviations: OS, Overall Survival; AJCC, American Joint Committee on Cancer; PCI, Propylactic Cranial Irradiation.

Through multigenic variant combination, these patients could be classified into three risk groups, which provided a basis to identify LD-SCLC patients who would benefit from chemoradiotherapy.

CASP7: rs4353229 was the most significant SNP associated with survival in these patients. Caspases are a family of cysteine-aspartic acid proteases that serve as initiators or executioners in cell apoptosis [17]. The CASP7 gene, located in exon 21 of chromosome $10 \mathrm{q} 25$, plays a crucial role during the execution phase of apoptosis. The loss of its expression is reportedly associated with a poor prognosis in renal cell carcinoma [18]. The CASP7 rs4353229 SNP was also determined to be predictive of survival in patients with advanced non- 
Table 2: Survival analysis of miRNA-related SNPs in apoptosis pathway genes

\begin{tabular}{|c|c|c|c|c|c|c|c|c|c|}
\hline Gene SNP & miRNA & \multicolumn{2}{|c|}{ Model } & $\begin{array}{l}5 y \\
\text { OS }\end{array}$ & HR $(95 \%$ CI) & $\begin{array}{c}\text { Log- } \\
\text { rank } \\
P\end{array}$ & $\begin{array}{c}\text { HR }(95 \% C I) \\
\text { (adjusted) * }\end{array}$ & $\begin{array}{c}P \\
\text { adjusted }\end{array}$ & $q$ \\
\hline \multirow{2}{*}{$\begin{array}{c}C A S P 8 \\
\text { rs } 1045494\end{array}$} & & \multirow[b]{2}{*}{ DOM } & ТT (90) & $28.7 \%$ & \multirow[b]{2}{*}{$\begin{array}{c}0.508 \\
(0.284-0.909)\end{array}$} & \multirow[b]{2}{*}{0.020} & \multirow[b]{2}{*}{$\begin{array}{c}0.514 \\
(0.287-0.921)\end{array}$} & \multirow[b]{2}{*}{0.025} & \multirow[b]{2}{*}{0.035} \\
\hline & & & $\begin{array}{c}\mathrm{TC}+\mathrm{CC} \\
(53)\end{array}$ & $55.7 \%$ & & & & & \\
\hline \multirow{2}{*}{$\begin{array}{c}\text { PIK3R1 } \\
\text { rs3756668 }\end{array}$} & \multirow{2}{*}{ miR-589-3p } & \multirow[t]{2}{*}{ REC } & $\begin{array}{c}\mathrm{GA}+\mathrm{GG} \\
(119)\end{array}$ & $35.5 \%$ & \multirow{2}{*}{$\begin{array}{c}0.417 \\
(0.190-0.916)\end{array}$} & \multirow[t]{2}{*}{0.025} & \multirow{2}{*}{$\begin{array}{c}0.421 \\
(0.190-0.931)\end{array}$} & \multirow[t]{2}{*}{0.033} & \multirow[t]{2}{*}{0.037} \\
\hline & & & $\mathrm{AA}(25)$ & $47.7 \%$ & & & & & \\
\hline \multirow{2}{*}{$\begin{array}{c}C A S P 7 \\
\text { rs4353229 }\end{array}$} & \multirow[t]{2}{*}{$\operatorname{miR}-520 a-5 p$} & \multirow[t]{2}{*}{ REC } & $\begin{array}{c}\mathrm{TC}+\mathrm{TT} \\
(125)\end{array}$ & $41.0 \%$ & \multirow{2}{*}{$\begin{array}{c}2.227 \\
(1.246-3.980)\end{array}$} & \multirow[t]{2}{*}{0.006} & \multirow{2}{*}{$\begin{array}{c}2.245 \\
(1.247-4.041)\end{array}$} & \multirow[t]{2}{*}{0.007} & \multirow[t]{2}{*}{0.023} \\
\hline & & & $\mathrm{CC}(21)$ & $24.1 \%$ & & & & & \\
\hline
\end{tabular}

*Adjusted for age, gender, Karnofsky performance score (KPS), smoking history, Charison comorbidity index (CCI) and propylactic cranial irradiation (PCI).

Abbreviations: SNP, single nucleotide polymorphism; OS, overall survival; HR: hazard ratio; CI, confidence interval.
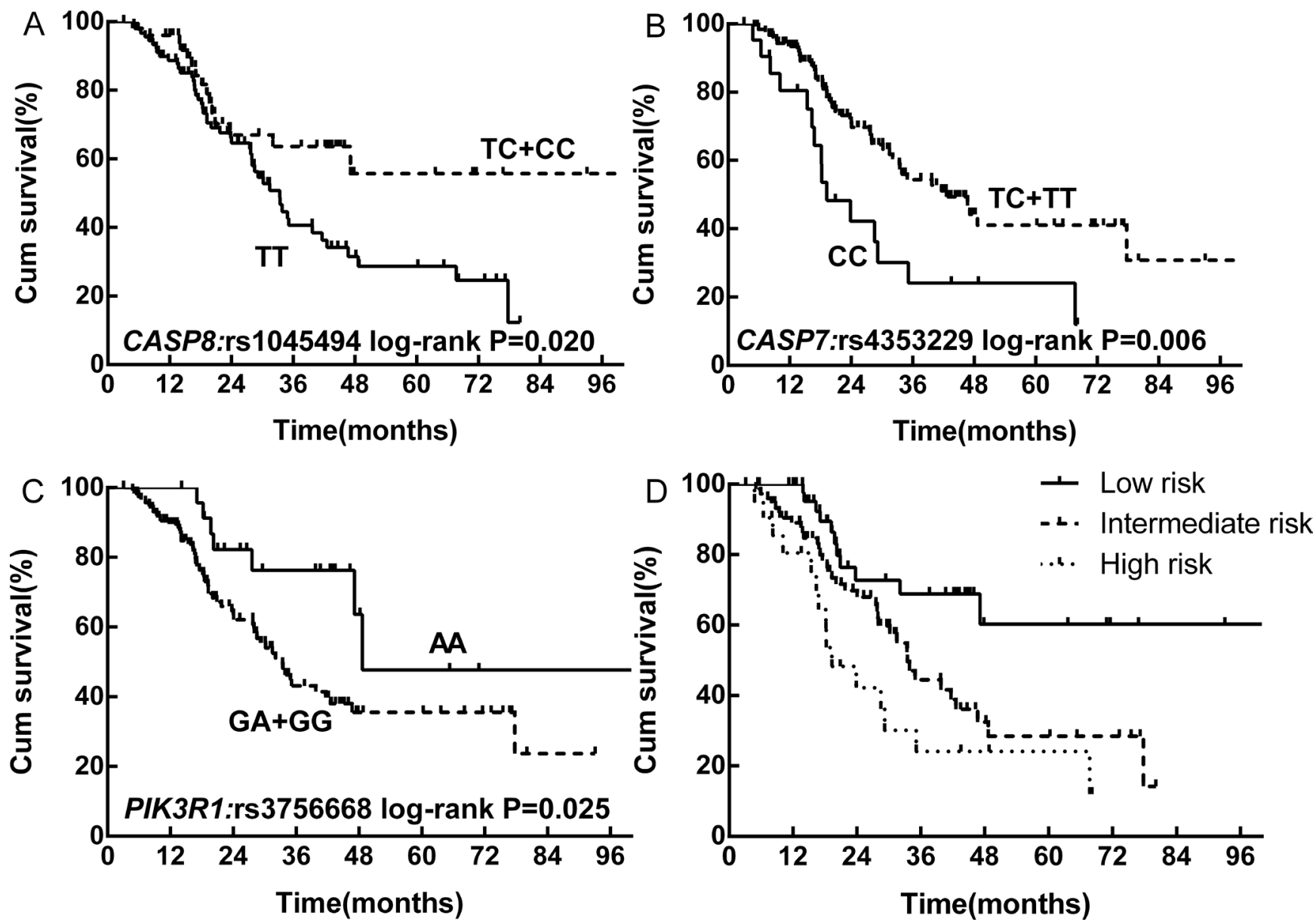

Figure 1: Kaplan-Meier survival curves for selected SNPs and the combined RPA classification in patients with LDSCLC treated with curative chemoradiotherapy. (A) CAP8: rs1045494. (B) CASP7: rs4353229. (C) PIK3R1: rs3756668. (D) RPA classification. 
Table 3: RPA classification based on unfavorable genotype combinations

\begin{tabular}{|c|c|c|c|c|c|c|c|}
\hline \multicolumn{2}{c}{ rs 4353229 } & rs1045494 & $n$ & 5y-OS & $\begin{array}{c}\text { HR (95\% CI) } \\
\text { adjusted }\end{array}$ & P adjusted* & P bootstrap \\
\hline Low risk & TT+TC & CC+TC & 48 & $60.3 \%$ & Ref & 0.005 & \\
\hline $\begin{array}{c}\text { Intermediate } \\
\text { risk }\end{array}$ & TT+TC & TT & 74 & $28.4 \%$ & $\begin{array}{c}2.150 \\
(1.081-4.275)\end{array}$ & 0.029 & 0.036 \\
\hline High risk & CC & Any & 21 & $24.1 \%$ & $\begin{array}{c}3.760 \\
(1.680-8.415)\end{array}$ & 0.001 & 0.007 \\
\hline
\end{tabular}

*Adjusted for age, gender, Karnofsky performance score (KPS), smoking history, Charison comorbidity index (CCI) and propylactic cranial irradiation (PCI).

Abbreviations: RPA, recursive partitioning analysis; OS, overall survival; HR, hazard ratio; CI: confidence interval.

small cell lung cancer [19]. However, there has been no direct functional study of rs4353229 variants. Instead, possible underlying mechanisms were inferred from data publically available online [20]. Rs4353229 C allele was reported to be associated slightly decreased levels of RNA expression in Asian population. On the other hand, the rs4353229 SNP is strongly linked to disequilibrium with the CASP7 rs2227309 SNP in the Chinese population $\left(r 2=0.98 ; \mathrm{D}^{\prime}=1.0\right)$, which is thought to influence the level of CASP7 mRNA expression and individuals' apoptotic capacity [21].

The CASP8: rs1045494 SNP was previously reported to be associated with breast cancer risk [22]. Caspase 8 is an apoptosis initiator in the extrinsic cell death pathway mediated by tumor necrosis factor (TNF) [23]. Soung et al. [24] demonstrated that somatic mutations decrease $C A S P-8$ activity during apoptosis as compared with wild-type. Notably, caspase- 8 expression is reportedly absent in SCLC, leading SCLC cells to be resistant to apoptosis $[25,26]$. In our study, the rs 1045494 $\mathrm{C}$ variant was significantly associated with a much better outcome than wild-type, suggesting the polymorphisms in the miRNA binding site enhanced the expression of caspase-8. Confirmation of the mechanism requires further investigation, however.

The A/A genotype of rs 3756668 , located in the 3'-UTR of PIK3R1, was associated with a considerable improvement in survival, though it did not enter the combined RPA model. PIK3RI is a component of the phosphoinositide-3 kinase (PI3K)-AKT-mammalian target of rapamycin (mTOR) pathway and encodes the $85-\mathrm{kD}$ regulatory subunit of $P I 3 K$. In vitro molecular investigation revealed that heterozygous disruption of PIK3RI decreased apoptosis induced by insulinlike growth factor 1 (IGF-1) through up-regulated phosphatidylinositol $(3,4,5)$-triphosphate production, while homozygous PIK3R1 significantly increased apoptosis reflecting a reduction in PI3-kinase activity. This is consistent with our analysis of the rs3756668 genotype (Supplementary Table S1) and suggests an effect of this SNP on PIK3R1 expression.
This is the first report on the association of SNPs affecting miRNA binding to targets in the apoptosis pathway on clinical outcome in SCLC. The adoption of a targeted pathway-based approach strengthens the effective power of the study and provides greater discrimination of outcomes with multigenic effects. Within the apoptotic signaling network, activated caspase-8 cleaves caspase-3 and/or caspase-7, initiating the apoptotic cascade [27]. Consistent with that, our findings show that the combined effect of the two SNPs in CASP8 and CASP7 is greater than that of the individual SNPs. However, the PIK3R1 SNP in another pathway could not be included in the final prognostic model. This may be due to the small number of SNPs selected in this in silico analysis, which potentially missed some key sites of interaction between different pathways and is a limitation of this study. Another limitation is that although this is the largest study yet conducted to explore miRNA-related SNPs associated with prognosis in SCLC, we did not recruit enough cases for independent validation. Finally, although evidence from previous studies support the biological plausibility of our findings, in vitro functional assays of the identified SNPs will be necessary determine the molecular mechanisms.

In sum, the present study showed that three SNPs affecting miRNA binding sites on components of the apoptosis pathway are prognostic in LD-SCLC patients treated with chemoradiotherapy. Moreover, a risk classification incorporating CASP8: rs 1045494 and CASP7: rs4353229 was developed to identify patients likely to benefit from treatment. Replication in large independent cohorts and determination of the molecular mechanism are imperative to confirm these findings.

\section{MATERIALS AND METHODS}

\section{Ethics statement}

This investigation was conducted in accordance with the ethical standards outlined in the Declaration of Helsinki and national and international guidelines. Our 
institutional review board (Cancer Hospital, Chinese Academy of Medical Sciences) approved this retrospective study and informed consent was waived.

\section{Study population}

Patients histologically confirmed as SCLC and initially treated at the Cancer Hospital, Chinese Academy of Medical Sciences between January 2007 and June 2014 were enrolled in this study. The inclusion criteria were as follows: (1) LD-SCLC staged based on the International Association of Lung Cancer (IASLC) classification [28]; (2) patients received curative-intent platin-based chemotherapy combined intensity-modulated radiation therapy (IMRT). Patients who had any other malignancy within 5 years of enrollment were excluded. Clinical information was collected from medical records. Long-term archived serum samples from these patients were analyzed [29].

\section{SNP selection and genotyping}

Candidate SNPs at miRNA-binding sites in the 3'-UTR of apoptotic pathway genes were selected using the PolymiRTS Database 3.0 (http://compbio.uthsc. edu/miRSNP/) and Ensemble Asia database (release 79, $\mathrm{http} / / /$ asia.ensembl.org/index.html? redirect $=$ no). SNPs in the apoptotic pathway previously reported in the literature to be associated with cancer were also included. Tagged SNPs in linkage disequilibrium were identified using HaploReg version 3 (http://www.broadinstitute.org/ mammals/haploreg/haploreg v3.php) with a cut-off value of $r^{2}>0.8$ and a minor allele frequency greater than 0.01 in the Chinese Han population.

DNA was extracted using a TIANamp Blood DNA Kit (TIANGEN BIOTECH, Beijing, China). Genotyping was conducted using a MALDI-TOF mass spectrometrybased iPLEX Gold assay on a Sequenom MassARRAY Platform (San Diego, CA, USA), and was analyzed using MassARRAY TyperAnalyzer v4.0 software (Sequenom). SNPs with a success rate of more than $95 \%$ and samples with a call rate exceeding $96 \%$ were included. Ultimately, a total of 12 tagSNPs in 7 genes were selected.

\section{Statistical analysis}

To ensure the reliability of the genotyping, the Hardy-Weinberg equilibrium was tested using the chisquare goodness-of-fit test with one degree of freedom. OS was calculated from pathologic diagnosis to the date of death or last follow-up. The survival estimates for each genotype and for clinical characteristics were calculated using the Kaplan-Meier method and compared using the log rank test. Hazard ratios (HRs) and 95\% confident intervals (CIs) were estimated using Cox proportional hazards regression models, adjusting for clinical characteristics associated with OS. All SNPs were analyzed in three genetic models (dominant, recessive and additive), and the most significant model was used. Models with a rare genotype observed $<5 \%$ percent of patients were excluded. Benjamini-Hochberg False Discovery Rate (FDR) correction was used for multiple testing with a $q$-value of 0.05 [30]. To evaluate the cumulative effects of the genetic variants in the pathway, recursive partitioning analysis (RPA) was conducted to combine the prognostic SNPs. The concordance probability estimate (CPE) was used to assess the predictive ability of the RPA classification [31] from 1000 bootstrap samples.

A two-sided $p$ values $<0.05$ was considered significant for all the statistical analyses. All the statistical analyses were carried out using IBM SPSS Statistics 21.0 software (IBM Corp., Armonk, NY) and R version 3.1.3 (www.R-project.org).

\section{ACKNOWLEDGMENTS}

None.

\section{CONFLICTS OF INTEREST}

All authors of this manuscript have no conflicts of interest to disclose.

\section{GRANT SUPPORT}

This work was supported by the National Nature Science Foundation of China (No. 81572971).

\section{REFERENCES}

1. Govindan R, Page N, Morgensztern D, Read W, Tierney R, Vlahiotis A, Spitznagel EL, Piccirillo J. Changing epidemiology of small-cell lung cancer in the United States over the last 30 years: analysis of the surveillance, epidemiologic, and end results database. J Clin Oncol. 2006; 24:4539-4544.

2. Pignon JP, Arriagada R, Ihde DC, Johnson DH, Perry MC, Souhami RL, Brodin O, Joss RA, Kies MS, Lebeau B, Onoshi T, Østerlind K, Tattersall MHN, et al. A metaanalysis of thoracic radiotherapy for small-cell lung cancer. N Engl J Med. 1992; 327:1618-1624.

3. Brambilla E, Negoescu A, Gazzeri S, Lantuejoul S, Moro D, Brambilla C, Coll JL. Apoptosis-related factors p53, Bcl2, and Bax in neuroendocrine lung tumors. Am J Pathol. 1996; 149:1941-1952.

4. Khan KH, Blanco-Codesido M, Molife LR. Cancer therapeutics: Targeting the apoptotic pathway. Crit Rev Oncol Hematol. 2014; 90:200-219.

5. Lowe SW, Lin AW. Apoptosis in cancer. Carcinogenesis. 2000; 21:485-495.

6. Fernandez-Cuesta L, Oakman C, Falagan-Lotsch P, Smoth KS, Quinaux E, Buyse M, Dolci MS, Azambuja ED, 
Hainaut P, Dell'orto P, Larsimont D, Francis PA, Crown J, et al. Prognostic and predictive value of TP53 mutations in node-positive breast cancer patients treated with anthracycline- or anthracycline/taxane-based adjuvant therapy: results from the BIG 02-98 phase III trial. Breast Cancer Res. 2012; 14:R70.

7. Xu P, Liu L, Wang JZ, Zhang K, Hong XH, Deng QF, Xiang JJ, Zhang XM, He MA, Wu TC, Guo H. Genetic Variation in BCL2 3 '-UTR Was Associated with Lung Cancer Risk and Prognosis in Male Chinese Population. Plos One. 2013; 8.

8. Knoefel LF, Werle-Schneider G, Dally H, Muller PJ, Edler L, Bartsch H, Tuengerthal S, Heussel CP, Reinmuth N, Thomas M, Risch A. Polymorphisms in the apoptotic pathway gene BCL-2 and survival in small cell lung cancer. J Thorac Oncol. 2011; 6:183-189.

9. Shukla GC, Singh J, Barik S. MicroRNAs: Processing, Maturation, Target Recognition and Regulatory Functions. Mol Cell Pharmacol. 2011; 3:83-92.

10. Yu Z, Li Z, Jolicoeur N, Zhang L, Fortin Y, Wang E, Wu M, Shen SH. Aberrant allele frequencies of the SNPs located in microRNA target sites are potentially associated with human cancers. Nucleic Acids Res. 2007; 35: 4535-4541.

11. Thompson CB. Apoptosis in the pathogenesis and treatment of disease. Science. 1995; 267:1456-1462.

12. Ashkenazi A. Targeting the extrinsic apoptotic pathway in cancer: lessons learned and future directions. J Clin Invest. 2015; 125:487-489.

13. Cotter TG. Apoptosis and cancer: the genesis of a research field. Nat Rev Cancer. 2009; 9:501-507.

14. Yang X, Gao F, Ma F, Ren Y, Chen H, Liang X, Han S, Xiong X, Pan W, Zhou C, Zhou L, Yang M. Association of the functional BCL-2 rs2279115 genetic variant and small cell lung cancer. Tumour Biol. 2016; 37:1693-8. doi: 10.1007/s13277-015-3934-9.

15. Lin J, Lu C, Stewart DJ, Gu J, Huang M, Chang DW, Lippman SM, Wu X. Systematic evaluation of apoptotic pathway gene polymorphisms and lung cancer risk. Carcinogenesis. 2012; 33:1699-1706.

16. Zhang F, Sturgis EM, Sun Y, Zhang Y, Wei Q, Zhang C, Zheng H, Li G. Apoptotic variants as predictors of risk of oropharyngeal cancer recurrence after definitive radiotherapy. Int J Cancer. 2015; 137:2454-2461.

17. Degterev A, Boyce M, Yuan J. A decade of caspases. Oncogene. 2003; 22:8543-8567.

18. Vilella-Arias SA, Rocha RM, da Costa WH, Zequi Sde C, Guimaraes GC, Verjovski-Almeida S, Soares FA, Reis EM. Loss of caspase 7 expression is associated with poor prognosis in renal cell carcinoma clear cell subtype. Urology. 2013; 82:974 e971-977.

19. Qian J, Gu S, Wu Q, Zhao X, Wu W, Gao Z, Zhang W, Tan X, Wang H, Wang J, Fan W, Chen H, Han B, et al. Association of CASP7 polymorphisms and survival of patients with non-small cell lung cancer with platinumbased chemotherapy treatment. Chest. 2012; 142:680-689.

20. Wang MY, Zhu ML, He J, Shi TY, Li QX, Wang YN, Li J, Zhou XY, Sun MH, Wang XF, Yang YJ, Wang JC, Jin L, et al. Potentially functional polymorphisms in the CASP7 gene contribute to gastric adenocarcinoma susceptibility in an eastern Chinese population. Plos One. 2013; 8:e74041.

21. Garcia-Lozano JR, Torres B, Fernandez O, Orozco G, Alvarez-Marquez A, Garcia A, Gonzalez-Gay MA, Garcia A, Nunez-Roldan A, Martin J, Gonzalez-Escribano MF. Caspase 7 influences susceptibility to rheumatoid arthritis. Rheumatology. 2007; 46:1243-1247.

22. Khan S, Greco D, Michailidou K, Milne RL, Muranen TA, Heikkinen T, Aaltonen K, Dennis J, Bolla MK, Liu J, Hall P, Irwanto A, Humphreys K, et al. MicroRNA related polymorphisms and breast cancer risk. Plos One. 2014; 9:e109973.

23. Danial NN, Korsmeyer SJ. Cell death: critical control points. Cell. 2004; 116:205-219.

24. Soung YH, Lee JW, Kim SY, Jang J, Park YG, Park WS, Nam SW, Lee JY, Yoo NJ, Lee SH. CASPASE-8 gene is inactivated by somatic mutations in gastric carcinomas. Cancer Res. 2005; 65:815-821.

25. Joseph B, Ekedahl J, Sirzen F, Lewensohn R, Zhivotovsky B. Differences in expression of pro-caspases in small cell and non-small cell lung carcinoma. Biochem Biophys Res Commun. 1999; 262:381-387.

26. Hopkins-Donaldson S, Ziegler A, Kurtz S, Bigosch C, Kandioler D, Ludwig C, Zangemeister-Wittke U, Stahel R. Silencing of death receptor and caspase- 8 expression in small cell lung carcinoma cell lines and tumors by DNA methylation. Cell Death Differ. 2003; 10:356-364.

27. Budihardjo I, Oliver $H$, Lutter $M$, Luo X, Wang X. Biochemical pathways of caspase activation during apoptosis. Annu Rev Cell Dev Biol. 1999; 15:269-290.

28. Micke P, Faldum A, Metz T, Beeh KM, Bittinger F, Hengstler JG, Buhl R. Staging small cell lung cancer: Veterans Administration Lung Study Group versus International Association for the Study of Lung Cancerwhat limits limited disease? Lung cancer. 2002; 37: 271-276.

29. Rounge TB, Lauritzen M, Langseth H, Enerly E, Lyle R, Gislefoss RE. microRNA Biomarker Discovery and High-Throughput DNA Sequencing Are Possible Using Long-term Archived Serum Samples. Cancer Epidemiol Biomarkers Prev. 2015; 24:1381-1387.

30. Benjamini Y, Hochberg Y. Controlling the False Discovery Rate - a Practical And Powerful Approach To Multiple Testing. J Roy Stat Soc B Met. 1995; 57:289-300.

31. Gonen M, Heller G. Concordance probability and discriminatory power in proportional hazards regression. Biometrika. 2005; 92:965-970. 